\title{
Ein Lacertilier (Scincomorpha, Paramacellodidae) aus dem Oberen Jura von Tendaguru (Tansania)
}

\author{
Annette Broschinski ${ }^{1}$
}

Mit 2 Abbildungen

\section{Zusammenfassung}

Seit einigen Jahren werden die Funde großer Wirbeltiere aus der oberjurassischen Fossilfundstelle Tendaguru in Tansania durch diverse Mikrovertebraten ergänzt. Diese konnten durch gezieltes Schlämmen von Sedimentmaterial gewonnen werden (Heinrich, mündl. Mitt.), das während der Tendaguru-Expeditionen von 1908 bis 1913 gewonnen wurde.

Durch ein isoliertes Kieferfragment kann der Erstnachweis eines paramacellodiden Lacertiliers im Afrika südlich des Äquators (bei $10^{\circ}$ südlicher Breite) geführt werden. Dieser Fund rundet das Bild der sehr weiten Verbreitung dieser erfolgreichen mesozoischen Echsengruppe ab.

Schlïsselwörter: Mesozoische Lacertilia, Paramacellodidae, Paläogeographie, Oberjura, Afrika.

\begin{abstract}
Recently, there have been additional microvertebrate finds within the known macrovertebrate fauna of the Upper Jurassic locality Tendaguru in Tanzania. This resulted from the processing of sediment samples, which had been collected during the Tendaguru Expeditions in between 1908 and 1913 (Heinrich, pers. Comm.).

An isolated jaw fragment from a paramacellodid lizard is the first record of this family within the African continent below the equator $\left(10^{\circ}\right.$ degrees Southern latitude). The occurence of this successful Mesozoic lizard group in Tendaguru reflects a greater global distribution than known to date.
\end{abstract}

Key words: Mesozoic Lacertilians, Paramacellodidae, Palaeogeography, Upper Jurassic, Africa.

\section{Einleitung}

Innerhalb der basalen Scincomorpha im Bereich Oberjura/Unterkreide aus verschiedensten Fundstellen dominieren die osteodermal gepanzerten Paramacellodiden, eine offensichtlich sehr erfolgreiche Familie. Diese ist mittlerweile für die Nordkontinente durch Funde recht gut belegt. Für die Südkontinente des ehemaligen Gondwana-Komplexes kann dieser Nachweis nun ebenfalls durch das unten beschriebene Fundstück erbracht werden. Dies ist insbesondere für das Verständnis der Ausbreitungsmechanismen von Bedeutung; die heute lebenden engsten Verwandten der Paramacellodiden, die Cordyliformes, sind auf die Südhalbkugel beschränkt (Afrika und Madagaskar). Zudem stellen sich die Paramacellodiden zunehmend als charakteristische Vergesellschaftung oberjurassischer Dino- saurierfundstellen heraus, auch wenn der vorliegende Einzelfund noch keine Aussagen über Häufigkeiten ermöglicht.

\section{Systematischer Teil}

Squamata Merrem, 1826

Scincomorpha Camp, 1923

Paramacellodidae Estes, 1983

Genus typicus: Paramacellodus Hoffstetter, 1967

Eingeschlossene Gattungen: Paramacellodus Hoffstetter 1967, Becklesius Estes 1983, Sharovisaurus Hecht \& Hecht 1984, Mimobecklesisaurus Li 1985.

Der Status von Pseudosaurillus, Saurillus und Saurillodon als zu den Paramacellodidae gehörig ist unsicher (Evans 1998), da ihre Überlieferung nur fragmentarisch ist.

\footnotetext{
${ }^{1}$ Niedersächsisches Landesmuseum Hannover, Naturkunde-Abteilung, Willy-Brandt-Allee 5, 30169 Hannover, Germany. Eingegangen April 1999, angenommen Juni 1999
} 
Diagnose der Paramacellodidae:

Die Paramacellodidae sind eine seit dem Mittleren Jura bis mindestens in die höhere Unterkreide auf der Nordhalbkugel weit verbreitete Gruppe von kleinen bis mittelgroßen Echsen mit einem generalisierten Bauplan und relativ kurzen, robusten Gliedmaßen.

Die Paramacellodidae werden hauptsächlich anhand von Schädelmerkmalen definiert (unpaares Prämaxillare, Parietale rechteckig mit schwacher vermiculater Skulpturierung, Vorhandensein eines Palpebralknochens in der oberen Orbita, hoher Dorsalfortsatz des Maxillare mit gleichzeitigem Ausgreifen des Medialfortsatzes in Richtung des gegenüberliegenden Maxillare, reduziertes oberes Temporalfenster mit großem Postorbitofrontale, und einige mehr: Estes 1983). Im Regelfall weisen sie eine Panzerung des gesamten Körpers durch rechteckige Osteoderme auf, die in einem flexiblen System gegenseitiger Überlappung (Imbrikation) eine hohe Beweglichkeit garantieren. Die Paramacellodidae besitzen kräftige Kiefer mit einer mit dem Individualalter wachsenden Mandibulartorsion (Hoffstetter 1967).

\section{Erweiterte Diagnose:}

Je nach Gattung ist eine Pterygomandibularis-Facette am Unterrand des Dentale ausgeprägt. Alle Gattungen weisen recht große Mentalformina auf. Die Zähne sind ebenfalls kräftig und auf einer verbreiterten Basis verankert. Sie sind leicht bauchig und tragen auf ihrer Spitze zwei aufeinander $\mathrm{zu}$ laufende Schneidekanten, die eine gemeinsame Spitze bilden (Cuspis labialis sensu Richter 1994). Die vordere Schneidekante ist stets deutlich länger. Auf der Innenseite ist direkt unterhalb dieser Spitze noch eine nach lingual weisende, zweite kleine Spitze ausgebildet (Cuspis lingualis sensu Richter 1994). Von den beiden Schneidekanten ziehen schwache Grate an den beiden Flanken der Zähne hinab, die leicht nach lingual weisen.

Die Zähne sind auf der Labialseite glatt, auf der Lingualseite durch ein charakteristisches Muster vorderer und hinterer Striationen gerieft. Diese Striae laufen ebenfalls zur Innenspitze hin aufeinander zu. Der Zahnersatz entspricht dem der Scincomorpha in Form kleiner, in der Mitte der Zahnbasis befindlicher Resorptionsgruben. Es sind etwa 20 bis maximal 26 Zähne auf dem Dentale ausgebildet.

Nach neueren Untersuchungen (Evans 1998) können die Paramacellodidae als Schwestergrup- pe der Scincidae und der Cordyliformes (sensu Lang 1991) angesehen werden. Vom Körperbau her müssen sie den heutigen afrikanischen und madegassischen Schildechsen sehr ähnlich gewesen sein (Gerrhosaurus, Zonosaurus, u. a.).

\section{Beschreibung des Fundstücks "Jg 824 / Mittlere Sauriermergel von Tansania"}

Das vorliegende, bisher einzige Fragment eines Lacertilier-Kiefers ist eine etwa $1 \mathrm{~mm}$ große Spitze eines linksseitigen Dentale. Das Stück ist offensichtlich leicht abgerollt; 3 Zähne sind jedoch erhalten.

In der Labialansicht (Fig. 1a) ist die relativ gut erhaltene Facies buccalis (sensu Rauscher 1992) zu erkennen. Ein großes, deutlich eingetieftes Mentalforamen liegt dicht am Ventralrand des Dentale. Eine Pterygomandibularis-Facette ist nicht zu erkennen, könnte jedoch auch direkt hinter der Bruchkante einsetzen. Eine leichte Unebenheit des Ventralrandes direkt hinter dem Foramen spricht für diese Interpretation.

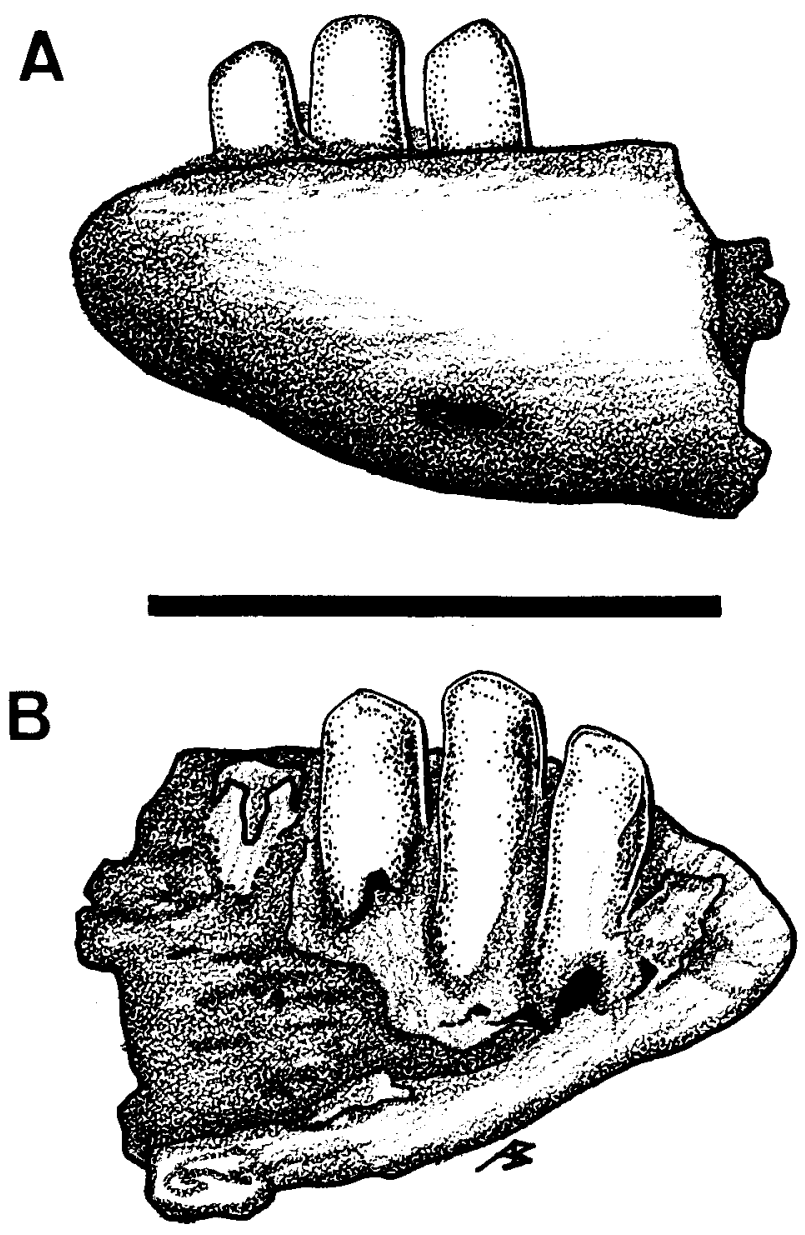

Fig. 1. Spitze eines linken Dentale (Jg. 824); 1A: Labialansicht, 1B: Lingualansicht. Maßstabsbalken: $1 \mathrm{~mm}$ 
Die drei erhaltenen Zähne zeigen in der Labialansicht die charakteristische glatte Außenseite sowie den kräftigen Schaft mit den beiden apicalen Schneidekanten, die die Zahnkrone bilden (Cuspis labialis). Die vordere Schneidekante ist jeweils länger als die hintere.

Auf der Lingualseite (Fig. 1b) ist von der Symphysenregion nicht mehr viel erhalten, der größte Teil des Knochens (subdentaler Schelf, ventrale Lamelle des Dentale, a.m.) ist stark zerstört. Die drei erhaltenen Zähne jedoch zeigen neben dem bereits von der Labialansicht bekannten Grundmuster der Schneidekanten auf ihren Innenseiten andeutungsweise Bildungen lingualer Spitzchen auf der Krone. Striae sind nicht zu erkennen, was jedoch auf den generell schlechten, abgerollt wirkenden Erhaltungszustand des Fragments zurückzuführen ist. Am vordersten Zahn ist jedoch eine Fortsetzung des Flankengrates zu erkennen, der von der vorderen Schneidekante des Zahnes nach ventral zieht. Ebenfalls am vordersten Zahn ist eine mittelgroße Resorptionsgrube zu erkennen.
Alle erhaltenen Merkmale sprechen eindeutig dafür, das Fundstück als Paramacellodidae indet. anzusprechen. Die auffällig breiten Schneidekanten, die beim vorliegenden Stück bereits weit vorn im Kiefer ausgeprägt sind, sprechen für eine vorläufige Zuordnung des Tandaguru-Fragments zur Gattung Becklesius. Die Gattung Paramacellodus weist eher lanzenförmige, spitze Zähne auf.

\section{Diskussion}

Die Bedeutung des vorliegenden Fundstückes liegt in zwei Informationen, in seiner stratigraphischen Position sowie seiner paläogeographischen Situation. Es handelt sich um den ersten Nachweis eines Paramacellodiden auf der Südhalbkugel, südlich des Äquators (bei ca. $10^{\circ}$ heutiger südlicher Breite; Fig. 2). Zudem repräsentiert das Stück den bisher ältesten nachweisbaren Vertreter dieser Familie auf dem afrikanischen Kontinent.

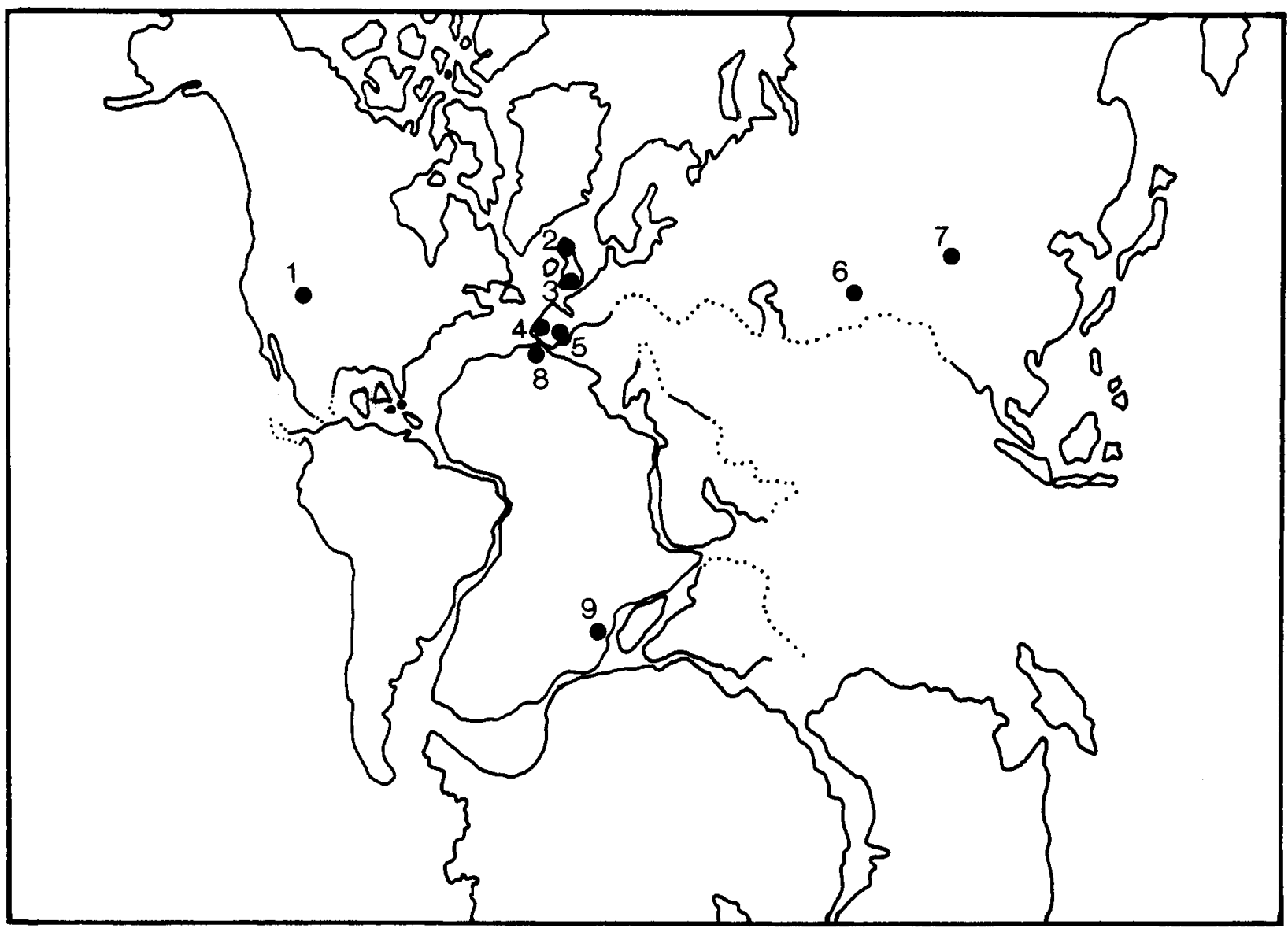

Fig. 2. Verbreitungskarte der Paramacellodidae in Jura und Unterkreide (Kontinentalkonfiguration entspricht dem Oberen Jura), Fundstellen markiert: 1 - Morrison Formation (Kimmeridgium), 2 - Skye/Schottland (Bathonium), 3 - Purbeck, Kirtlington (Berriasium, Bathonium), 4 - Guimarota (Kimmeridgium), 5 - Uña und Galve (Barremium), 6 - Karatau/ Kasachstan (Kimmeridgium), 7 - Gansu, China ("Oberjura"), 8 - Anoual/Marokko (?Berriasium), 9 - Tendaguru/Tansania (Oxfordium, Kimmeridgium). Nach Evans (1993, 1998), verändert 
Neben Seiffert (1973), Estes (1983) und Richter (1994) hat sich insbesondere Evans (1993, 1996, 1998) intensiv mit den mesozoischen Squamata, u. a. den Paramacellodidae, befaßt. In ihren jüngsten Beschreibung paramacellodider Echsen aus der nordamerikanischen Morrison-Formation (Evans 1998, Evans \& Chure 1998) gibt sie einen kurzen Abriß der Verbreitung der Paramacellodidae, die lange Zeit als auf die Nordhalbkugel beschränkt angenommen wurde. In einer unterkretazischen Fauna Marokkos konnten jedoch paramacellodide Dentalia und Osteoderme nachgewiesen werden (Gattung Paramacellodus: Richter, 1994; Broschinski \& Sigogneau-Russell 1996; ?Berriasium). Der nun erfolgte Nachweis der Verbreitung von Paramacellodidae bis weit in einen Südkontinent hinein vervollständigt das Bild der Paramacellodidae als erfolgreiche und zudem global weit verbreitete Gruppe, die zeitlich deutlich früher einsetzt, als bisher vermutet wurde. Zudem bietet er bessere Erklärungsmöglichkeiten für das Schwestergruppenverhältnis $\mathrm{zu}$ den rezenten Cordyliformes, die in ihrer Verbreitung ausschließlich auf Afrika und Madagaskar beschränkt sind. $\mathrm{Zu}$ welchem Zeitpunkt die Trennung beider Linien stattgefunden hat, ist nicht bekannt. Aufgrund der lückenhaften Fundsituation in Afrika kann derzeit noch nicht über das tatsächliche Ausbreitungszentrum der Paramacellodidae geurteilt werden; Wanderbewegungen sind sowohl von der Nordhalbkugel nach Afrika als auch umgekehrt vorstellbar. Es steht zu hoffen, daß weitere Untersuchungen im bereits vorhandenen Sauriermergel-Material, vielleicht ergänzt durch neues Material, weitere Funde ermöglichen werden.

\section{Danksagung}

Meinen herzlichsten Dank möchte ich Herrn Dr. W.-D. Heinrich, Berlin, aussprechen, der mich auf das Fundstück aufmerksam machte, es zur Bearbeitung zur Verfügung stellte und zudem neu hinzukommendes Material zur kritischen Durchsicht zugänglich machte. Herrn Prof. Dr. H. P. Schultze, Berlin, gilt mein Dank für die allzeit mögliche Sammlungsarbeit.

Großer Dank gebührt Frau Dr. S. Evans, London, die mich aufs großzügigste mit Ihren aktuellen Separata ausstattete.

Herrn Prof. Dr. B. Krebs, Berlin, möchte ich ebenfalls für zusätzliche Literatur und die kritische Durchsicht des Manuskriptes danken, ebenso wie einem unbekannten Revisor.

\section{Literatur}

Broschinski, A. \& Sigogneau-Russell, D. 1996. Remarkable lizard remains from the Lower Cretaceous of Anoual (Morocco). - Annales de Paléontologie (Vert.-Invert.), 82 (3): 147-175.

Estes, R. 1983. Sauria terrestria, Amphisbaenia. - Handbuch der Paläoherpetologie, Part 10A, XXII+249 pp., 69 Abb.; Gustav Fischer Verlag, Stuttgart.

Evans, S. E. 1993. Jurassic Lizard Assemblages. - Revue de Paléobiologie, Vol. spéc. 7: 55-65.

- 1996. Parviraptor (Squamata: Anguimorpha) and other lizards from the Morrison Formation at Fruita, Colorado. Continental Jurassic Symposium Volume, Museum of Northern Arizona, Bulletin 60: 243-248.

- 1998. Paramacellodid lizard skulls from the Jurassic Morrison Formation at Dinosaur National Monument, Utah. - Journal of Vertebrate Paleontology 18 (1): 99-114.

Evans, S. E. \& Chure, D. 1998. Morrison lizards: Structure, relationships and biogeography. - Modern Geology 23: $35-48$.

Hoffstetter, R. 1967. Coup d'oeil sur les sauriens (= lacertiliens) des Couches de Purbeck (Jurassique supérieur d'Angleterre). - Colloques Internationaux du Centre $\mathrm{Na}$ tional de la Recherche Scientifique 163: 349-371.

Lang, M. 1991. Generic relationships within Cordyliformes (Reptilia: Squamata). - Bulletin van het Koninklijk Belgisch Instituut voor Natuurwetenschapen, Biologie 61: 121-188.

Rauscher, K. L. 1992. Die Echsen (Lacertilia, Reptilia) aus dem Plio-Pleistozän von Bad Deutsch-Altenburg, Niederösterreich. - Beiträge zur Paläontologie von Österreich 17: $81-177$.

Richter, A. 1994. Lacertilia aus der Unteren Kreide von Uña und Galve (Spanien) und Anoual (Marokko). - Berliner geowissenschaftliche Abhandlungen (E) 14: 1-147.

Seiffert, J. 1973. Upper Jurassic lizards from Central Portugal. - Servicos Geológicos de Portugal, Memória 22: $1-85$. 